\title{
Frecuencia de las señales de alarma en pacientes con cefalea, atendidos en la emergencia de un hospital de Lima.
}

\author{
Frequency of red flags in patients with headaches treated in an emergency unit in Lima.
}

Xavier A. Santander ${ }^{1, a}$, Mario C. Rivera ${ }^{1, a}$, Martín A. Tipismana ${ }^{1,2, b}$

\section{RESUMEN}

Las cefaleas en emergencia suelen ser un reto diagnóstico para el evaluador cuando se trata de diferenciarlas entre primaria o secundaria a un trastorno neurológico subyacente. Objetivo: Describir la frecuencia de las señales de alarma en cefaleas secundarias y primarias en pacientes que acuden a emergencia. Materiales y Métodos: Estudio prospectivo de series de casos, que acuden a la Emergencia de Medicina de Adultos del Hospital Nacional Cayetano Heredia por cefalea, en un mes. Se excluyeron a los pacientes con cefalea traumática. Se usó un formato para la obtención de datos. Se clasificó a los pacientes en cefalea primaria o secundaria y se determinó la presencia de señales de alarma. Resultados: Se registraron 32 pacientes. Veintiséis (81\%) tuvieron cefalea secundaria. De éstas, 9 se debieron a causas infecciosas y 9 a trastornos vasculares intracraneales. La cantidad de señales de alarma presentes en las cefaleas secundarias vario de 3 a 8, con una moda de 5; y en las primarias de 0 a 2, con una moda de 2. El empeoramiento progresivo y el examen neurológico anormal se presentaron en $96 \%$ de los casos de cefalea secundaria. Conclusiones: Las señales de alarma, empeoramiento progresivo y examen neurológico anormal fueron los más frecuentes. Las cefaleas secundarias fueron más comunes que las cefaleas primarias. Se hace énfasis en la utilidad de usar esta metodología en emergencia.

PALABRAS CLAVE: Cefalea, emergencia, señales de alarma.

\section{SUMMARY}

Headaches in the emergency room are usually a diagnostic challenge for the physician when they having to differentiate between a primary and secondary headaches and an underlying neurological disorder. Objectives: Describe the frequency of presentation of "red flags" in primary and secondary headaches, in patients presenting to an emergency department. Methods: A case-series prospective study was conducted in the Emergency room of Hospital Nacional Cayetano Heredia. All patients presenting with headaches within a month were evaluated. Trauma related headaches were excluded. A data collection form was used to determine the presence of red flags as well as other clinical data. The quantity of red flags was assessed in both types of headaches. Results: Thirtytwo patients were included for study. Twenty-six $(81 \%)$ had secondary headaches. Nine of them were found to be secondary to infection and other 9 to intracranial vascular disorders. The quantity of red flags present on secondary headaches ranged from 3 to 8 with a mode of 5 ; on primary headaches they ranged from 0 to 2 with a mode of 2 . Progressive worsening of headache and abnormal neurologic exam were present in 25 cases of secondary headache

1. Universidad Peruana Cayetano Heredia. Lima, Perú.

2. Hospital Nacional Cayetano Heredia. Lima, Perú.

a. Médico.

b. Médico neurólogo. 
Conclusions: Red flags were progressive worsening of headache and abnormal neurologic exam. Thus the clinical assessment of red flags is useful in the evaluation of patients with secondary headache in the emergency room.

KEY WORDS: Headache, emergency, warning signs.

\section{INTRODUCCIÓN}

El abordaje del paciente con cefalea en los servicios de emergencia, suele poner al clínico en la disyuntiva de considerar la cefalea como síntoma primario o secundario a un trastorno neurológico subyacente que cuando no es bien diagnosticado o identificado oportunamente, puede poner en peligro la vida del paciente. Para diferenciar las cefaleas, la literatura sugiere identificar las señales o características de alarma (SA) $(1,2)$, los cuales son hallazgos de la anamnesis y del examen físico, relacionados a un mayor riesgo de morbi-mortalidad. La utilidad de estas señales de alarma ha sido estudiada en otras realidades. Rueda Sánchez y col. en Colombia las encontraron en $93 \%$ de las cefaleas secundarias (3).

Las cefaleas representan del 1\% al 3\% del total de admisiones a emergencia en diferentes partes del mundo $(5,6)$, describiéndose un $2,2 \%$ de admisiones en un servicio de emergencia en Boston (7), pero también puede alcanzar un 9,49\%, como lo demuestra un estudio colombiano (3). La prevalencia de cefaleas primarias y secundarias en emeregencia, difieren en varios estudios. Por ejemplo, para cefaleas primarias Luda (8) encontró una frecuencia de 56\%, Bigal (9) reportó 77\% y Friedman (10) el 64\%. La International Classification of Headache Disorders, Segunda Edición, Primera Revisión (ICHD-IIr1), define cefalea secundaria como aquella que ocurre de novo conjuntamente con otro desorden reconocido como causante de cefalea (11). El uso de la ICHD-IIr1 en emergencia tiene un uso restringido. Como señala Friedman, la ICHD-IIr1 en emergencia ayuda a una clasificación reproducible de cefaleas, sin embargo, en un tercio de los casos no es de utilidad.

Las señales de alarma son diversas. Por ejemplo, se describe que la cefalea por tumor cerebral está presente en el $60 \%$ de los casos y es un síntoma solitario en el $2 \%$ de casos, presentando característica de empeoramiento matutino y con las maniobras de Valsalva (13). La cefalea por hemorragia subaracnoidea se ha descrito clásicamente como súbita e intensa, y se encuentra entre $45-97 \%$ de los casos $(14,15)$, es persistente en el $47 \%$ y se presenta con rigidez de cuello entre el $35-66 \%$ de admisiones
$(14,16)$. En cuanto a cefalea por trombosis cerebral venosa, puede acompañarse de déficit focal en 27 $68,9 \%$, crisis convulsiva en $10-61 \%$ y papiledema en $27-85 \%$ como se reportan en algunas series (17). Como se observa, estas características pueden orientar a un diagnóstico etiológico y oportuno.

\section{MATERIALES Y MÉTODOS}

Se realizó un estudio comparativo de 2 series de casos, prospectivo de pacientes con diagnóstico de cefalea, como síntoma principal o acompañante, que acudieron al Departamento de Emergencia, Servicio de Medicina de Adultos del Hospital Nacional Cayetano Heredia, Lima, Perú, entre el 6 de enero al 6 de febrero del 2011. Se incluyeron de forma no aleatoria consecutiva pacientes ambulatorios (estancia $<$ de 6 horas) como hospitalizados. Se incluyeron pacientes mayores de 14 años que llegaban al servicio por cefalea, ya sea como síntoma principal o acompañante; los criterios de exclusión fueron: a) pacientes cuya cefalea fue por causa traumática y b) aquellos que se negaron a ser entrevistados. La recolección de datos se realizó por parte de los investigadores a través de una entrevista directa con todos los pacientes. Se revisó la historia clínica, de donde se registraron los hallazgos del examen físico realizado preferentemente por el neurólogo o en su defecto por el médico residente de emergencia, así como los resultados de análisis de líquido cefalorraquídeo e informes de tomografía. Dentro del examen físico no se tomaron datos sobre papiledema ya que ningún paciente fue sometido a fundoscopía por parte del personal de emergencia. Todos estos datos fueron registrados en un formato adaptado para el estudio. Los investigadores no influenciaron en el diagnóstico. Clasificamos a los pacientes como cefaleas primarias y secundarias utilizando la clasificación vigente de la ICHD-IIr1 (11), en base al diagnóstico realizado por el neurólogo, o por el residente de emergencia.

Cefalea primaria se define como aquella que cumple los criterios de migraña, cefalea tensional o cefalea en racimos. Cefalea secundaria se define como aquella cefalea de novo con un desorden subyacente reconocido como factor causal de cefalea. Se empleó la lista de señales de alarma consignada en el trabajo 
Frecuencia de las señales de alarma en pacientes con cefalea, atendidos en la emergencia de un hospital de Lima.

Tabla 1: Listado de señales de alarma consignados.

1. Edad $>50$ años

2. Cefalea de instalación brusca

3. Empeoramiento progresivo de la cefalea

4. Empeora con esfuerzos físicos, tos o pujo

5. Antecedente de VIH y/o Neoplasia

6. Empeora con cambios de posición

7. Manifestaciones asociadas*

8. Signos vitales anormal al examen

9. Examen neurológico anormal**

10. Pérdida de equilibrio

11. Edema de papila

*Alteración de nivel de conciencia, fiebre, debilidad, vómitos explosivos, convulsiones, diplopía, disartria, ataxia.

** Rigidez de nuca, anisocoria, paresia, reflejos anormales y/o asimétricos.

Adaptado de Randall C. Evaluation of acute headache in adults. Am. Fam. Phys 2001; 63(4), 685-692. de Randall et. al. (12) (Tabla 1). Asimismo, se solicitó la colaboración de los pacientes y al aceptar se les pidió firmar un consentimiento informado.

El análisis estadístico se realizó utilizando medidas paramétricas de tendencia central como mediana, media y moda según la naturaleza de las variables, y de dispersión como desviación estándar y rango cada vez que fue necesario. Se procesaron los datos con el software Microsoft Excel 2010.

\section{RESULTADOS}

Durante el periodo de estudio se presentaron 32 pacientes con cefalea como síntoma principal o acompañante. De éstos, 6 (19\%) pacientes tuvieron cefalea primaria y $26(81 \%)$ secundaria. La edad promedio en el grupo de cefaleas primarias fue 41 años y en las secundarias fue 40 años. Diecisiete pacientes (53\%) fueron de sexo masculino, de los cuales 14

Tabla 2: Características demográficas y clínicas.

\begin{tabular}{|c|c|c|}
\hline $\begin{array}{l}\text { Total de pacientes } \\
\mathrm{n}=32(100 \%)\end{array}$ & $\begin{array}{l}\text { Cefaleas Primarias } \\
n=6\end{array}$ & $\begin{array}{l}\text { Cefaleas Secundarias } \\
\mathrm{n}=26\end{array}$ \\
\hline \multicolumn{3}{|l|}{ Edad } \\
\hline $\begin{array}{l}16-79 \text { años } \\
\text { DS: } 39,96 \pm 17.23\end{array}$ & $\begin{array}{l}23-79 \text { años } \\
41,16 \pm 21.49\end{array}$ & $\begin{array}{l}16-68 \text { años } \\
(39,69 \pm 16,59)\end{array}$ \\
\hline \multicolumn{3}{|l|}{ Sexo } \\
\hline Masculino:17 (53) & $3(50)$ & $14(53,85)$ \\
\hline Femenino:15 (47) & $3(50)$ & $12(46,15)$ \\
\hline \multicolumn{3}{|l|}{ Tipo de admisión } \\
\hline Ambulatorios: $7(21,8)$ & $6(100)$ & $1(4,34)$ \\
\hline Hospitalizados:25 $(78,2)$ & 0 & $25(95,66)$ \\
\hline \multicolumn{3}{|l|}{ Cefalálgico habitual } \\
\hline $\begin{array}{l}\text { Sí } \\
13(40,65)\end{array}$ & $4(67)$ & $9(34,61)$ \\
\hline $\begin{array}{l}\text { No } \\
19(59,45)\end{array}$ & $2(33)$ & $17(65,39)$ \\
\hline \multicolumn{3}{|l|}{ Tipo de dolor } \\
\hline $\begin{array}{l}\text { Pulsátil } \\
7(21,88)\end{array}$ & $3(50)$ & $4(15,38)$ \\
\hline $\begin{array}{l}\text { Opresivo } \\
12(37,5)\end{array}$ & $2(33,33)$ & $10(38,46)$ \\
\hline $\begin{array}{l}\text { Hincada } \\
11(34,37)\end{array}$ & $1(16,67)$ & $10(38,46)$ \\
\hline $\begin{array}{l}\text { Quemante } \\
2(6,25)\end{array}$ & 0 & $2(7,7)$ \\
\hline
\end{tabular}


Santander X, et al.

Tabla 2: Características demográficas y clínicas (continuación).

\begin{tabular}{|c|c|c|}
\hline $\begin{array}{l}\text { Total de pacientes } \\
\mathrm{n}=32(100 \%)\end{array}$ & $\begin{array}{l}\text { Cefaleas Primarias } \\
n=6\end{array}$ & $\begin{array}{l}\text { Cefaleas Secundarias } \\
\mathrm{n}=26\end{array}$ \\
\hline $\begin{array}{l}\text { Intensidad del dolor } \\
1-10 \\
(\text { mediana }=8)\end{array}$ & $\begin{array}{l}3-9 \\
(\text { mediana }=7)\end{array}$ & $\begin{array}{l}1-10 \\
(\text { mediana }=8)\end{array}$ \\
\hline $\begin{array}{l}\text { Duración del dolor } \\
0,01-21 \text { días } \\
(4,46 \text { días })\end{array}$ & $\begin{array}{l}0,25 \text { - } 4 \text { días } \\
\text { (1,5 días) }\end{array}$ & $\begin{array}{l}0,01-21 \text { días } \\
\text { (5,14 días) }\end{array}$ \\
\hline \multicolumn{3}{|l|}{ Nivel de conciencia } \\
\hline $\begin{array}{l}\text { Lúcido } \\
21(65)\end{array}$ & $6(100)$ & $15(57,7)$ \\
\hline $\begin{array}{l}\text { Confuso } \\
5(16)\end{array}$ & 0 & $5(19,23)$ \\
\hline $\begin{array}{l}\text { Hipersomne } \\
6(19)\end{array}$ & 0 & $6(23,07)$ \\
\hline \multicolumn{3}{|l|}{ Examen físico } \\
\hline $\begin{array}{l}\text { Normal } \\
7(21,88)\end{array}$ & $6(100)$ & $1(3,85)$ \\
\hline $\begin{array}{l}\text { Anormal } \\
25(78,12)\end{array}$ & 0 & $25(96,15)$ \\
\hline
\end{tabular}

Tabla 3: Exámenes realizados en los pacientes del estudio $(\mathrm{n}=32)$.

\begin{tabular}{|c|c|c|c|c|c|}
\hline \multicolumn{2}{|c|}{$\begin{array}{c}\text { Exámenes realizados } \\
(\%)\end{array}$} & \multicolumn{2}{|c|}{$\begin{array}{c}\text { Cefaleas primarias } \\
\mathrm{n}=6(\%)\end{array}$} & \multicolumn{2}{|c|}{$\begin{array}{c}\text { Cefaleas secundarias } \\
n=26(\%)\end{array}$} \\
\hline \multicolumn{6}{|l|}{ Punción Lumbar } \\
\hline \multirow{2}{*}{$\begin{array}{c}\text { Sí } \\
9(28,13)\end{array}$} & Normal & \multicolumn{2}{|c|}{0} & \multicolumn{2}{|l|}{$1(3,8)$} \\
\hline & Anormal & \multicolumn{2}{|c|}{0} & \multicolumn{2}{|c|}{$8(30,8)$} \\
\hline \multicolumn{2}{|l|}{$\begin{array}{c}\text { No } \\
23(71,87)\end{array}$} & \multicolumn{2}{|c|}{$6(100)$} & \multicolumn{2}{|c|}{$17(65,4)$} \\
\hline \multicolumn{6}{|l|}{ TC cerebral } \\
\hline \multirow{2}{*}{$\begin{array}{c}\text { Sí } \\
17(53,12)\end{array}$} & \multirow{2}{*}{$\begin{array}{l}\text { Normal } \\
\text { Anormal }\end{array}$} & \multicolumn{2}{|c|}{$1(17)$} & \multicolumn{2}{|c|}{$3(11,54)$} \\
\hline & & \multicolumn{2}{|c|}{0} & \multicolumn{2}{|l|}{$13(50)$} \\
\hline \multicolumn{2}{|l|}{$\begin{array}{c}\text { No } \\
15(46,88)\end{array}$} & \multicolumn{2}{|c|}{$5(83)$} & \multicolumn{2}{|c|}{$10(38,46)$} \\
\hline \multirow{2}{*}{\multicolumn{2}{|c|}{ Exámenes }} & \multicolumn{4}{|c|}{ Cefaleas } \\
\hline & & & Primarias & Secundarias & Iotal \\
\hline \multirow{3}{*}{ Punción lumbar } & \multirow{2}{*}{$\mathrm{Si}$} & Normal & 0 & 1 & 1 \\
\hline & & Anormal & 0 & 8 & 8 \\
\hline & No & & 6 & 17 & 23 \\
\hline \multirow{3}{*}{ Tomografía crane } & \multirow{2}{*}{$\mathrm{Si}$} & Normal & 1 & 3 & 4 \\
\hline & & Anormal & 0 & 13 & 13 \\
\hline & No & & 5 & 10 & 15 \\
\hline
\end{tabular}


Frecuencia de las señales de alarma en pacientes con cefalea, atendidos en la emergencia de un hospital de Lima.

Tabla 4: Clasificación de cefaleas secundarias y primarias halladas en el estudio según la ICHD-IIr1

\begin{tabular}{|c|c|c|}
\hline & Pacientes & Porcentaje \\
\hline Cefaleas primarias & 6 & 100 \\
\hline Migraña & 3 & 50 \\
\hline Tensional & 3 & 50 \\
\hline Cefaleas secundarias & 26 & 100 \\
\hline Trastorno vascular craneal o intracraneal & 9 & 34,61 \\
\hline Enfermedad cerebro- vascular & 5 & 19,20 \\
\hline Hemorragia subaracnoidea & 4 & 15,41 \\
\hline Infecciones & 9 & 34,61 \\
\hline Meningitis bacteriana & 4 & 15,41 \\
\hline Meningitis tuberculosa & 3 & 11,53 \\
\hline Meningitis criptococócica & 2 & 7,67 \\
\hline Trastorno No vascular intracraneal & 7 & 26,92 \\
\hline Síndrome vertiginoso & 1 & 3,91 \\
\hline Neoplasia intracraneal & 2 & 7,67 \\
\hline Síndrome convulsivo & 2 & 7,67 \\
\hline Hipertensión intracraneana & 2 & 7,67 \\
\hline Trastornos de la homeostasis & 1 & 3,84 \\
\hline Gastroenterocolitis aguda con deshidratación moderada + hipokalemia & 1 & 3,84 \\
\hline
\end{tabular}

$(53,5 \%)$ presentaron cefalea secundaria. Todos los pacientes con cefalea primaria fueron atendidos ambulatoriamente y 25 pacientes $(95,6 \%)$ con cefalea secundaria fueron hospitalizados. En el grupo de cefaleas primarias $4 / 6(67 \%)$ tuvieron historia de cefalea y en las secundarias 9/26 (34,6\%). El tipo de dolor más común en el grupo de cefaleas primarias fue pulsátil $(\mathrm{n}=3 ; 50 \%)$ y en las secundarias el opresivo (10/26) e hincada (10/26). La intensidad del dolor, tuvo una mediana de 8 para cefaleas secundarias y 7 para cefaleas primarias. El promedio de duración del dolor fue de 5,1 días para el grupo de cefaleas secundarias y de 1,5 días para el grupo de primarias.

Respecto al nivel de conciencia al ingreso, en el grupo de cefaleas secundarias el $57,7 \%(\mathrm{n}=15)$ estuvo lúcido mientras que el $23 \%(n=6)$ estuvo hipersomne y el 19,2\% $(n=5)$ confuso. Todos los pacientes del grupo de cefaleas primarias estuvieron lúcidos. En cuanto al examen físico el 78,1\% $(n=25)$ fue anormal en la serie, representando estos el $96.1 \%$ del grupo de cefaleas secundarias. En las primarias, todos tuvieron examen físico normal (Tabla 2).
Se realizó punción lumbar para estudio de líquido cefalorraquídeo en 9 pacientes $(34,6 \%)$ del grupo de cefaleas secundarias, resultando anormales $8(30,8 \%)$. De igual forma, 16 pacientes $(61,5 \%)$ del grupo de cefaleas secundarias fue sometido a una tomografía cerebral, de los cuales $13(50 \%)$ resultaron anormales (Tabla 3). Solo 1 paciente del grupo de cefaleas primarias fue sometido a tomografía, resultando normal.

En cuanto a la etiología, según la ICHDIIr1, para el grupo de primarias, $3(50 \%)$ fueron migraña y 3 $(50 \%)$ tensionales. En el grupo de las secundarias, los trastornos más comunes fueron los vasculares intracraneales $(n=9)$, infecciosas $(n=9)$ y los no vasculares intracraneales $(n=7)$ (Tabla 4).

Las señales de alarma por paciente en el grupo de cefaleas primarias tuvo una moda de 2 y el grupo de secundarias tuvo una moda de 5 (Gráfico 1). En el grupo de cefaleas primarias, la señal de alarma más común fue el de manifestaciones asociadas a cefalea $(50 \%)$. En las cefaleas secundarias los más comunes, 
Gráfico 1: Frecuencia de señales de alarma en cefaleas primarias y

$$
\text { secundarias }
$$

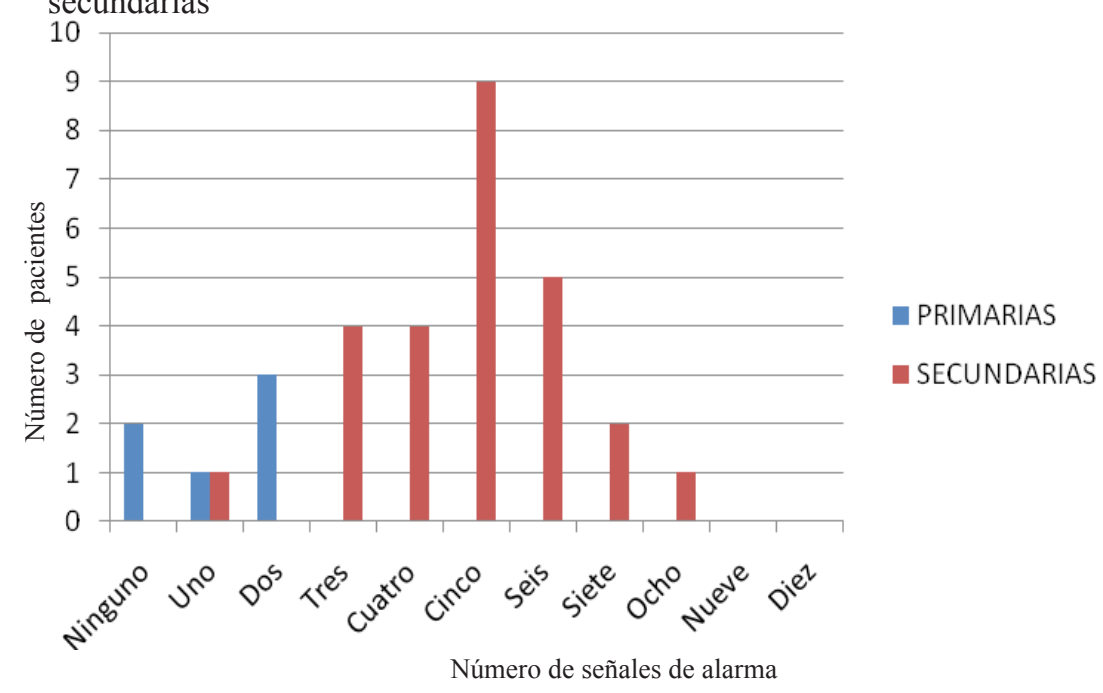

Tabla 5: Frecuencia total de señales de alarma en cefaleas primarias y secundarias.

\begin{tabular}{lcc}
\hline \multicolumn{1}{c}{$\begin{array}{c}\text { Signo de Alarma } \\
\mathrm{n}=149(100 \%)\end{array}$} & $\begin{array}{c}\text { Cefaleas } \\
\text { primarias } \\
\mathrm{n}=6(\%)\end{array}$ & $\begin{array}{c}\text { Cefaleas } \\
\text { secundarias } \\
\mathrm{n}=26(\%)\end{array}$ \\
\hline Examen neurológico anormal *25 (16,7) & 0 & $25(96,1)$ \\
Empeoramiento progresivo 26 $(17,44)$ & $1(16,6)$ & $25(96,1)$ \\
Manifestaciones asociadas a cefalea **26 $(17,44)$ & $3(50)$ & $23(88.4)$ \\
Empeora con cambios de posición20 $(13,4)$ & 0 & $20(76,9)$ \\
Empeora con maniobra de Valsalva13 $(8,7)$ & 0 & $13(50)$ \\
Antecedente de VIH y/o Neoplasia10 $(6,71)$ & 0 & $10(38,4)$ \\
Instalación brusca10 (6,71) & $1(16,6)$ & $9(34,6)$ \\
Signos vitales anormales 7 (4,69) & 0 & $7(26,9)$ \\
Edad $>50$ años8 $(5,3)$ & $1(16,6)$ & $7(26,9)$ \\
Pérdida de equilibrio6 (4) & 0 & $6(23)$ \\
\hline
\end{tabular}

Un paciente podía tener uno o más señales de alarma de forma concomitante.

* Paresia, rigidez de nuca, alteración del nivel de conciencia.

** Debilidad, vómitos y convulsiones

en forma decreciente, fueron: examen neurológico anormal (96,1\%) (alteración del nivel de conciencia, paresia y rigidez de nuca), empeoramiento progresivo $(96,1 \%)$, manifestaciones asociadas a cefalea $(88,4 \%)$ (debilidad, vómitos y convulsiones), empeoramiento con cambios de posición $(76,9 \%)$, maniobras de Valsalva $(50 \%)$ y antecedente de VIH y/o neoplasia $(38,4 \%)$ (Tabla 5).

\section{DISCUSIÓN}

Durante el periodo de recolección de datos, se atendieron 2042 pacientes en el servicio de emergencia de medicina. Los 32 pacientes de nuestra serie representan el 1,3\% de éstos. Al respecto, sobre la prevalencia de cefaleas en los servicios de emergencia, no se cuentan con datos nacionales y a 
Frecuencia de las señales de alarma en pacientes con cefalea, atendidos en la emergencia de un hospital de Lima.

nivel de Latinoamérica son escasos. Por ejemplo en Brasil, Bigal (9) encontró que en un centro primario fue 9,3\% y en Colombia, Rueda-Sánchez (3), encontró el 9,49\%. En países industrializados la prevalencia de cefaleas en emergencia, varía entre $1-4 \%(1,5,6,7$, 20,21). Los resultados encontrados estarían dentro del rango esperado considerando que nuestra institución es un hospital general.

En relación a las cefaleas secundarias estas fueron más frecuentes en nuestro estudio ( $81 \%$ vs. $19 \%)$. Este resultado difiere con otras publicaciones, en las que las cefaleas primarias suelen ser las predominantes. Locker (4) en Inglaterra encontró $42,1 \%$ de cefaleas secundarias, Luda (8) en Italia un 44\% y Bigal (9) en Brasil un 33\%. Nuestros datos se aproximan a los encontrados por Rueda-Sánchez (3) en Colombia (75\%). Esta diferencia puede deberse al nivel socioeconómico de la población y la diferente prevalencia de cefaleas primarias, así como al tipo de hospital donde se realizó el estudio. En nuestra serie, la mayor frecuencia de cefaleas secundarias es comparable con la de Rueda-Sánchez (3) y puede deberse a diversos factores: nuestro hospital es un centro de referencia, es un hospital general y posiblemente una mayor frecuencia de infecciones del sistema nervioso central.

La etiología más común de cefaleas secundarias fueron los trastornos vasculares intracraneales $(9$ pacientes) y las infecciones del sistema nervioso central (9 pacientes), que en otros estudios representaron porcentajes minoritarios $(0,6-2,9 \%)(4,19)$.

En cuanto a la intensidad del dolor, que fue más alta en el grupo de cefaleas secundarias, es importante aclarar que no existe una relación directa en cuanto a intensidad y gravedad de cefalea, ya que algunas cefaleas primarias como la migraña pueden ser muy intensas.

Las señales de alarma fueron más frecuentes en las cefaleas secundarias (Tabla 5), en orden fueron: empeoramiento progresivo de cefalea, examen neurológico anormal y manifestaciones asociadas a cefalea. Por otro lado en el estudio prospectivo de Locker et. al., se determinaron tres factores predictivos de patología secundaria: edad $>50$ años, cefalea de inicio súbito y examen neurológico anormal. La presencia de manifestaciones asociadas a cefalea (vómitos, fiebre y convulsiones) ya ha sido demostrada como predictor de patologías serias, con un LR (+) de 1,41 (4). El examen neurológico anormal (alteración del nivel de conciencia, paresia y rigidez de nuca) es una constante que se repite tanto en nuestro estudio como en otros $(4,6,18)$ lo que corrobora su importancia para identificar cefaleas secundarias.

El número de señales de alarma por paciente también fue mayor en las cefaleas secundarias (rango: 3 - 8, moda: 5) respecto a las cefaleas primarias (rango: $0-2$, moda: 2). Aunque en la literatura no se encuentran datos sobre correlación entre número de señales de alarma y predicción de cefalea secundaria, esta metodología actualmente es el mejor método para poder identificarlas (12). Se debe señalar que $4 / 6$ pacientes con cefalea primaria presentaron al menos un signo de alarma, lo que demuestra la complejidad del diagnóstico diferencial en emergencia (Tabla 5).

Existen hallazgos del examen físico cuya presencia aumentan la probabilidad de encontrar resultados anormales en un examen de imágenes. De los pacientes con cefalea secundaria 16 fueron sometidos a tomografía y 9 a punción lumbar. El 50\% tuvo hallazgo de imagen anormal (infarto, edema y lesiones tumorales). Esto demuestra que la utilización de exámenes auxiliares de manera costo-efectiva, es importante en pacientes con cefalea secundaria ya que son la base del enfoque diagnóstico y terapéutico.

Finalmente, una de nuestras limitaciones está relacionada con la muestra reducida de pacientes. Consideramos que puede existir un subregistro de pacientes puesto que no pudieron captarse todos los casos. También debemos explicar que los diagnósticos en la sala de emergencia pudieron verse influenciados por el nivel de experiencia del examinador, no necesariamente especialista.

En nuestro estudio, las cefaleas secundarias fueron más frecuentes que las primarias, y dentro de este grupo las atribuidas a infecciones y trastornos vasculares intracraneales son las más importantes. La búsqueda de señales de alarma en pacientes con cefalea en una emergencia es una herramienta vital para identificar diagnósticos ominosos, en este estudio las más frecuentes el empeoramiento progresivo, examen neurológico anormal, manifestaciones asociadas a cefalea, empeoramiento con cambios de posición y maniobras de Valsalva.

Es recomendable que los médicos en emergencia usen rutinariamente las señales de alarma para diferenciar las cefaleas secundarias de las primarias. 


\section{Correspondencia:}

Dr. Martín Tipismana

e-mail:martintipismana@yahoo.com.mx

\section{REFERENCIAS BIBLIOGRÁFICAS}

1. Toledo J B, Riverol M, Martinez-Vila P, Irimia P. Cefalea en urgencias. AnSistSanitNavar 2008; 31:1.

2. Gauvrit JY, Leclerc X, Moulin T, Oppenheim C, Savage J, Pruvo JP, Meder JF. Céphalées dans un contexte d'urgence. J Neuroradiol. 2004; 31: 262270.

3. Rueda-Sánchez M, Mantilla F, Solano M. Prevalencia de cefaleas en un servicio de urgencias en Colombia. Rev Neurol 2005; 40:209-213.

4. Locker E, Thompson C, Rylance J, Mason SM. The Utility of Clinical Features in Patients Presenting With Nontraumatic Headache: An Investigation of Adult Patients Attending an Emergency Department. Headache 2006; 46:954-961.

5. Fontanarosa PB. Recognition of subarachnoid hemorrhage. Ann Emerg Med 1989; 18(11):1199205.

6. Agostoni E. Headache in cerebral venous thrombosis. Neurol Sci 2004; 25:S206-S210.

7. Goldstein JN. Camargo CA, Pelletier AJ, Edlow JA. Headache in United States emergency departments: demographics, work-up and frequency of pathological diagnoses. Cephalalgia 2006; 26:684690.

8. Luda E. The symptom of headache in emergency departments: the experience of a neurology emergency department. Ital J Neurol Sci 1995; 16:295-301.

9. Bigal M. Headache in an emergency room in Brazil. Sao Paulo Med J 2000; 118:58-62.

10. Friedman BW, Hochberg ML, Esses D, Grosberg $\mathrm{B}$, Corbo J, Toosi BM et al. Applying the International Classification of Headache Disorders to the Emergency Department: An Assessment of Reproducibility and the Frequency With Which a Unique Diagnosis Can Be Assigned to Every Acute Headache Presentation. Ann Emerg Med2007; 49(4):409-19.
11. Headache Classification Subcommittee. The international classification of headache disorders. Cephalalgia 2004; 24(suppl.1):1-160.

12. Randall C. Evaluation of Acute headache in Adults. Am. Fam. Phys 2001; 63(4), 685-692.

13. Schankin CJ. Ferrari U. et al. Characteristics of brain tumour-associated headache. Cephalalgia 2007; 27:904-911.

14. Barton CV. Evaluation and treatment of headache patients in the emergency department: a survey. Headache 1994; 34:91-94.

15. Weir B. Headaches from aneurysms. Cephalalgia 1994; 14:79-87.

16. Gorelick PB, Hier DB, Caplan LR, Langenberg P. Headache in acute cerebrovascular disease. Neurology 1986; 36(11):1445-50.

17. Guenther G, Arauz A. Trombosis venosa cerebral: aspectos actuales del diagnóstico y tratamiento. Neurología. 2011; 26(8):488-498.

18. Ramirez-Lassepas M, Espinosa CE, Cicero JJ, Johnston KL, Cipolle RJ, Barber DL. Predictors of intracranial pathologic findings in patients who seek emergency care because of headache. Arch Neurol.1997; 54:1506-1509.

19. Mert E, Özge A, Tasdelen B, Yilmaz A, Bilgin N. What clues are available for differential diagnosis of headaches in emergency settings? J Headache Pain 2008; 9:89-97.

20. Edlow J, Caplan L. Avoiding pitfalls in the diagnosis of subarachnoid hemorrhage. NEJM 2000; 342:2936.

21. Edlow J, Panagos P, Godwin S, Thomas T, Decker W. Clinical Policy: Critical Issues and Management of Adult Patients presenting to the emergency department with acute headache. Ann EmergMed 2008; 52(4):407-36.

Recibido: 24/04/2013

Aceptado: 12/06/2013 